\title{
As covariantes da confiança política na América Latina
}

\author{
Célia Mara Ladeia Colen \\ Programa de Pós-Graduação em Ciência Política \\ Universidade Federal de Minas Gerais
}

\begin{abstract}
Resumo: As abordagens culturalistas, baseadas nos fatores relacionados com a socialização, e institucionalistas, baseadas nos fatores relacionados à experiência com o sistema político, competem pela explicação dos fatores relacionados aos baixos níveis de confiança política na América Latina. Este artigo faz uma breve revisão das duas perspectivas, compara seus poderes preditivos para explicar a confiança política na região. A partir do Latinobarômetro 2005 , aplicou-se a técnica do modelo hierárquico linear com o objetivo de considerar a dependência entre as observações no nível do país. A confiança política é maior entre os que apóiam o presidente; entre aqueles que estão em países considerados mais democráticos; entre os indivíduos que avaliam positivamente a situação econômica, as políticas públicas e a capacidade do governo para combater a corrupção e entre os indivíduos com maior sofisticação política. As variáveis culturais, por sua vez, quais sejam, a confiança interpessoal e a avaliação dos concidadãos também aumentam a confiança política, mas têm efeito muito menor do que as variáveis institucionais.
\end{abstract}

Palavras-chave: instituições democráticas; confiança política; abordagem culturalista; abordagem institucionalista; modelo hierárquico linear

\begin{abstract}
Culturalist approaches, based on factors related to socialization, and institutionalist one, based on factors related to experience with the political system, compete for the explanation of the factors related to low levels of political trust in Latin America. This paper briefly reviews these two approaches and compares their predictive power to explain political trust in the region. Using data from Latinobarometro 2005, the technique of hierarchical linear model it was applied with the purpose of considering the dependence among observations at country level. The political trust is higher among those who support the president; among those who are in countries that are considered the most democratic; individuals who positively evaluate the economic situation, public policies and government capacity to combat corruption; individuals with greater political sophistication. The cultural variables, interpersonal trust and the assessment of co-citizens also enhance political trust, but have much smaller effect than the institutional variables.
\end{abstract}

Keywords: democratic institutions; political trust; culturalist approach; institutionalist approach; hierarchical linear model 


\section{Introdução ${ }^{1}$}

A confiança nas instituições políticas tem sido um fenômeno de grande interesse na Ciência Política desde a segunda metade do século XX. O estudo foi retomado no final dos anos oitenta nas democracias estabelecidas com o objetivo de compreender se a queda da confiança nas instituições representava risco para o regime democrático. Nos países que passavam pela transição à democracia naquele mesmo período, o tema também despertou interesse, buscando-se saber as possibilidades de consolidação das democracias recém-estabelecidas dada a desconfiança dos cidadãos de suas instituições. Assim, nas últimas décadas, países da África, do Leste Europeu e da América Latina têm sido objetos de crescentes pesquisas de opinião para avaliar as atitudes dos cidadãos em relação ao regime democrático.

0 estudo dos fatores relacionados à confiança nas instituições tem suas raízes na abordagem culturalista iniciada pelos estudos de Almond e Verba (1963) e David Easton (1965). Nessas obras, os fatores relacionados com a socialização mostraram-se centrais na explicação das atitudes dos indivíduos em relação à política, mas a experiência com o regime também foi um elemento considerado. Um aspecto que tem sido resgatado dessa tradição pelas pesquisas culturalistas recentes, como em Lagos (2000) e Power \& Jamison (2005), é que a confiança política teria origem nos processos de socialização, podendo ser considerada como uma extensão da confiança interpessoal. Importantes críticas têm sido dirigidas a essa abordagem por autores da perspectiva institucionalista. Levi \& Stoker (2000) ressaltam que a questão central é avaliar se as instituições se apresentam como confiáveis para os indivíduos e Miller \& Listhaug (1999) defendem que o desempenho do regime é o elemento central para explicar a confiança política.

Nos estudos sobre a América Latina, um dos poucos consensos na literatura é que os níveis de confiança são baixos, mesmo quando comparados aos níveis de alguns países africanos em processo de transição para a democracia. Várias questões estão abertas ao debate: como operacionalizar o conceito de confiança política? Quais são as variáveis explicativas do fenômeno? Quais consequências podem ser derivadas dos baixos níveis de confiança? E, por fim, estão em debate as questões sobre o significado teórico da confiança ${ }^{2}$. Considerando a amplitude do dissenso, que não é característica específica da literatura latinoamericana, este artigo avalia criticamente a operacionalização do conceito de confiança política e compara o poder preditivo das variáveis culturalistas e institucionalistas. Para isso, apresenta uma análise das covariantes da confiança nas instituições democráticas

\footnotetext{
${ }^{1}$ Agradeço aos comentários dos pareceristas anônimos da Revista Opinião Pública.

2 Moisés (2005b) apresenta uma síntese dos trabalhos que tratam da relação entre confiança e democracia.
} 
na América Latina, buscando evidenciar os fatores comuns na região. $\mathrm{O}$ artigo orienta-se pela crítica, dirigida à abordagem culturalista, de que não é possível estudar a confiança política sem levar em conta a experiência dos indivíduos, posterior à socialização com o regime político. Outra contribuição é a aplicação da técnica de análise multinível através do modelo hierárquico linear utilizada neste artigo de modo exploratório com o objetivo de reconhecer a dependência entre as observações recolhidas em um mesmo país e avaliar a importância do contexto para a confiança política.

A análise exploratória das covariantes da confiança nas instituições democráticas apresentou resultados similares aos obtidos por pesquisas feitas no campo institucionalista e por pesquisas que buscam uma associação entre esta vertente teórica e a abordagem culturalista. $\mathrm{O}$ apoio ao presidente (variável usada como proxi da identificação com o partido no poder) está associado com o aumento da confiança nas instituições. Além disso, as variáveis relacionadas ao contexto importam: em países considerados mais democráticos e nos quais o nível de corrupção é considerado menor, a confiança é maior. No nível individual, as variáveis cognitivas e de avaliação das políticas também são importantes: os indivíduos mais sofisticados politicamente e que avaliam positivamente as políticas públicas confiam mais nas instituições. As variáveis culturais também mantiveram correlação razoável com a confiança política. No entanto, contribuíram pouco quando comparadas com o efeito das variáveis institucionais.

\section{O estudo da confiança política}

A investigação da confiança política nas democracias consolidadas sofreu uma importante inflexão a partir dos anos oitenta. Os estudos coordenados por Norris (1999) e por Pharr \& Putnam (2000) desafiaram os prognósticos de crise da democracia produzidos por Crozier et al (1975). Essa revisão foi orientada pelo aprofundamento da noção de David Easton de que o apoio político é multidimensional: variando das formas difusas (apoio à nação e apoio ao regime) às formas específicas de apoio (confiança nas instituições, satisfação com o funcionamento da democracia, confiança nas autoridades políticas). Partindo da constatação de que esses objetos de apoio têm definições distintas, teórica e empiricamente, os trabalhos reunidos por Norris (1999) constataram que a diminuição dos níveis de apoio específico (confiança nas autoridades, nas instituições e satisfação com o regime) não oferecia perigo para a democracia. Duas expressões foram cunhadas para caracterizar os indivíduos que são capazes de distinguir o funcionamento das instituições dos atributos do regime político: "cidadãos críticos" e "democratas insatisfeitos". Em vez de representarem uma 
ameaça à democracia, esses cidadãos deveriam ser considerados um instrumento para o aprimoramento do regime.

Na América Latina, os estudos sobre a confiança política têm forte influência da abordagem culturalista. No entanto, distanciando-se da combinação entre causas históricas e causas relacionadas ao desempenho dos sistemas políticos, presente nos estudos de Almond \& Verba (1963) e Easton (1965), a ênfase das análises culturalistas é posta nas causas históricas. Lagos (2000) é uma referência desses estudos - a autora defende que "características culturais pré-racionais e nãoracionais" configurariam as bases da cidadania latinoamericana. Os traços socioculturais baseados na prática de "permanecer em silêncio com relação aos seus sentimentos [...] e enfatizar as aparências" teriam sido parte da estratégia de sobrevivência dos indivíduos sob o colonialismo ibérico (LAGOS, 2000, p.2). Esse traço da cultura teria se enraizado no modo de viver dos cidadãos latinoamericanos, estruturando uma convivência marcada pela desconfiança entre as pessoas e, por decorrência, pela desconfiança em relação às instituições do Estado. Analisando os resultados do Latinobarômetro de 1996, a autora concluiu que a democracia na região, comparada aos padrões das democracias estabelecidas, enfrentaria um cenário problemático de baixos níveis de confiança interpessoal e pouca confiança dos cidadãos na sua capacidade de influenciar as decisões políticas.

Power \& Jamison (2005) consideram a "síndrome cultural" de desconfiança generalizada indicada por Lagos como o principal fator para explicar a desconfiança dos cidadãos em relação aos políticos e às instituições políticas democráticas. Esse quadro poderia levar a um círculo vicioso: em um primeiro momento, a desconfiança política leva a um comportamento antipartido por parte das elites; os resultados eleitorais positivos dessas elites não se sustentariam no médio prazo, levando ao poder governos que não conseguem cumprir as promessas feitas; no longo prazo, o processo levaria à exacerbação da desconfiança política. É interessante notar que os fatores relativos ao desempenho econômico e institucional são introduzidos pelos autores para explicar a baixa confiança política na região, mas a relação desses fatores com os culturais não é elaborada.

A explicação da confiança política voltada apenas para elementos do passado não permite vislumbrar o grande desafio enfrentado pela região. A dimensão desse desafio, que é comum a todas as nações que compuseram a terceira onda de democratização, é destacada por Norris: esses países teriam de enfrentar a constituição do Estado-nação e, simultaneamente, dar conta de reformar as estruturas econômicas e políticas. Rose et al (1999) ressaltam que, de maneira geral, a distância entre a democracia tomada como um ideal e a sua realidade seria inevitável; nos países recém-democratizados essa tensão seria ainda maior. Enquanto as nações democráticas estabelecidas alcançaram o equilíbrio através de processos incrementais de capacitação do Estado para a inclusão social, nas novas 
democracias, demandas crescentes seriam direcionadas para estruturas institucionais pouco aparelhadas. As estratégias de tentativa e erro dos políticos provocam nos cidadãos uma impressão de que os regimes são muito dispendiosos e pouco eficazes. Essa tensão potencial é o contexto das novas democracias e o seu reconhecimento pode permitir um entendimento mais adequado dos baixos níveis de confiança na região.

A consideração dos desafios enfrentados por qualquer nova democracia é o ponto de partida necessário para evitar que a região seja tratada como um caso especial ou desviante em relação às outras experiências democráticas. Além do esforço comparativo, outra perspectiva que orienta este artigo é aquela que trata as teorias institucionalistas e culturalistas como complementares. Retomando importante aspecto da tradição dos estudos em cultura política, Mishler \& Rose (2001) destacam que as duas perspectivas teóricas consideram a experiência dos indivíduos como o elemento central para a compreensão da confiança política. Enquanto a perspectiva culturalista enfatiza as experiências pré-políticas do período da socialização, a perspectiva institucionalista destaca a experiência dos indivíduos com as instituições políticas na fase adulta. Os autores defendem a integração das duas vertentes no lifetime learning model. Nesse modelo, a confiança interpessoal aprendida na socialização pode ser projetada na confiança política, mas a vivência com as instituições políticas pode tanto reforçar quanto modificar essa experiência anterior. Para os autores, tanto os fatores associados à cultura política quanto os fatores relativos à avaliação do desempenho das instituições são relevantes para explicar a confiança política.

Mas os elementos contextuais são fundamentais para entender como se dá a relação de confiança entre cidadãos e instituições. Jong-Sung (2005) destaca que a corrupção e a desigualdade são fatores importantes para explicar as baixas taxas de confiança social. O autor cita Margaret Levi para destacar que instituições que são capazes de monitorar as leis e punir aqueles que estão fora da lei criam um ambiente mais propício para o aumento da confiança social. O mesmo processo pode ocorrer em relação à confiança política: em um ambiente marcado pela desigualdade acentuada, como é a América Latina, a percepção de que o sistema político e o sistema econômico beneficiam diferencialmente os indivíduos pode levar a uma sensação de que as instituições não são confiáveis. Por outro lado, a percepção de que o regime democrático está avançando pode criar um ambiente de confiança institucional. Braton (2002) evidencia que os bens políticos (eleições livres, competição política, direitos individuais, avaliação positiva das políticas governamentais) importam mais do que os bens econômicos para o apoio à democracia. Por fim, também é esperado que se vive em um ambiente democrático aumente $o$ apoio às instituições do regime. 


\section{A operacionalização da confiança política e as variáveis independentes}

O primeiro desafio para a análise da confiança política na América Latina está na operacionalização do conceito. A maioria dos estudos busca uma aproximação do índice produzido por Norris (1999), que utiliza a confiança nas instituições do Estado. Rovner (2005) construiu um índice de "apoio às instituições democráticas" a partir das instituições que ela considerou intrinsecamente relacionadas com o regime: governo, parlamento, judiciário, forças armadas, polícia e partidos políticos. Lopes (2004) trabalha com um índice somatório a partir dos itens de confiança na Presidência da República, no Congresso Nacional e nos partidos políticos. Nas duas medidas a inclusão da confiança no governo pode confundir o apoio difuso, relativo às instituições democráticas, com o apoio específico, representado pela confiança nas autoridades políticas e no governo eleito.

Neste artigo, a variável dependente é a confiança nas instituições democráticas e, para isso, construiu-se um índice de confiança a partir de quatro questões: confiança no Congresso, confiança nos partidos políticos, confiança na administração pública e confiança no judiciário ${ }^{3}$. Não se incluiu a confiança no Presidente e a confiança no governo para evitar a confusão entre as duas dimensões do apoio político. O conceito de multidimensionalidade defendido por Norris tem a vantagem de distinguir a confiança nas pessoas que estão no poder da confiança institucional, não sendo adequado, portanto, juntar itens de dois níveis distintos de apoio em apenas um indicador. Questões relativas à confiança nas forças armadas e na polícia não foram incluídas porque essas variáveis poderiam dificultar a separação entre indivíduos que confiam em instituições democráticas e aqueles que aumentam sua pontuação no índice porque têm alta confiança no exército instituição associada ao passado autoritário da região ${ }^{4}$.

Como variáveis de segundo nível, que caracterizarão as diferenças entre os países, utilizou-se o grau de democracia percebida e o nível de corrupção percebida. Essas variáveis visam captar quão democrático e quão corrupto os indivíduos consideram seu regime. As variáveis independentes, de primeiro nível, incluídas na análise, visam comparar o poder preditivo das vertentes culturalistas e institucionalistas. As variáveis relativas às teorias culturalistas incluídas na análise são a confiança interpessoal, a satisfação com a vida e a avaliação que se faz dos concidadãos. Espera-se que os indivíduos que confiam nas outras pessoas, que estão satisfeitos com a vida e que consideram seus concidadãos cívicos

\footnotetext{
${ }^{3}$ Verificar no Apêndice 2 as informações sobre o processo de criação do índice.

${ }^{4}$ Além disso, a ausência do exército na Costa Rica implicaria a exclusão desse país da amostra.
} 
(cumpridores da lei, cientes das obrigações e exigentes dos seus direitos) tenham mais confiança nas instituições ${ }^{5}$.

Para a definição das variáveis relativas à teoria institucionalista incluídas no modelo, algumas assertivas dessa abordagem foram consideradas. MCallister (1999) indica importantes diferenças entre os fatores relacionados com a confiança política em democracias novas e em democracias estabelecidas. Para este autor, nas novas democracias, a satisfação econômica é mais importante para a confiança nas instituições do que nas democracias estabelecidas, assim como, haveria uma ligação maior entre a confiança no governo e a confiança nas instituições do regime. Essa associação não ocorreria do mesmo modo nas democracias estabelecidas porque a frequência de eleições teria gerado um reservatório de apoio às instituições democráticas, o que levaria os cidadãos a distinguir claramente entre as instituições do Estado, os partidos no poder e os líderes eleitos em cada pleito.

Mas a capacidade de distinguir esses diferentes níveis e entidades de poder não impede que as opiniões dos indivíduos em relação à força política que está no poder influenciem o nível de confiança nas instituições. Para Norris (1999), os indivíduos que votaram nos políticos que estão no poder, os chamados "ganhadores", tenderiam a considerar as instituições mais responsivas às suas demandas, enquanto os "perdedores" seriam menos confiantes. Bowler \& Donovan (2003) concluíram que os eleitores que não elegeram seu candidato na eleição presidencial dos Estados Unidos de 2000 tinham menor confiança na Suprema Corte e no governo federal do país.

Neste artigo, utilizou-se a aprovação do presidente como uma proxi da variável "identificação com o partido no poder" para testar a hipótese de que os chamados "ganhadores" têm maior confiança nas instituições. Outra hipótese a ser testada é a de que existe um efeito independente da avaliação do desempenho das instituições sobre o aumento da confiança. Por isso, variáveis relativas aos aspectos cognitivos dos cidadãos e à percepção da situação econômica e política serão incluídas. A sofisticação política ${ }^{6}$ e a avaliação das políticas buscam captar elementos da experiência política dos cidadãos nos períodos pós-socialização. As variáveis relativas ao desempenho do regime são: a avaliação da situação econômica do indivíduo, a percepção do combate à corrupção e a avaliação do avanço das políticas públicas. A hipótese é que os mais sofisticados e os mais satisfeitos com o desempenho têm maior confiança nas instituições.

\footnotetext{
${ }^{5}$ Não foi possível utilizar o tamanho da cidade porque não são todos os países que têm amostra representativa das populações rural e urbana.

6 Segue-se o conceito de sofisticação política desenvolvido por Neuman (1985) e adaptado por Castro (1994).
} 


\section{Dados e metodologia}

Os dados utilizados neste artigo são provenientes do Latinobarômetro $2005^{7}$. O survey é composto por 20.209 entrevistas aplicadas em 18 países da região; o número de entrevistas por país varia entre 1.000 e $1.200^{8}$. Este é um estudo exploratório que utiliza o modelo hierárquico linear para avaliar as covariantes da confiança política, e procura contribuir com o desafio de considerar fatores contextuais na análise ${ }^{9}$.

Neste artigo, as opiniões dos indivíduos sobre as instituições de seu país compõem os dados de primeiro nível, dependentes entre si. Essas opiniões dependem do funcionamento das instituições avaliadas, contempladas nos elementos de segundo nível.

A variável de segundo nível é uma agregação da avaliação dos indivíduos em relação ao grau de democracia do seu país ${ }^{10}$. Outra variável agregada no nível do país foi a percepção da corrupção: Agregou-se em uma média para o país, as avaliações individuais de quantos funcionários públicos são corruptos.

A agregação das informações individuais nesse segundo nível de análise permitiu avaliar os efeitos das variáveis relativas ao nível individual, controlados pelos níveis de democracia e de corrupção percebidas. A classificação feita por especialistas foi testada como variável de segundo nível, mas ela não se mostrou eficiente para explicar a variação da confiança entre os países ${ }^{11}$. Para os objetivos

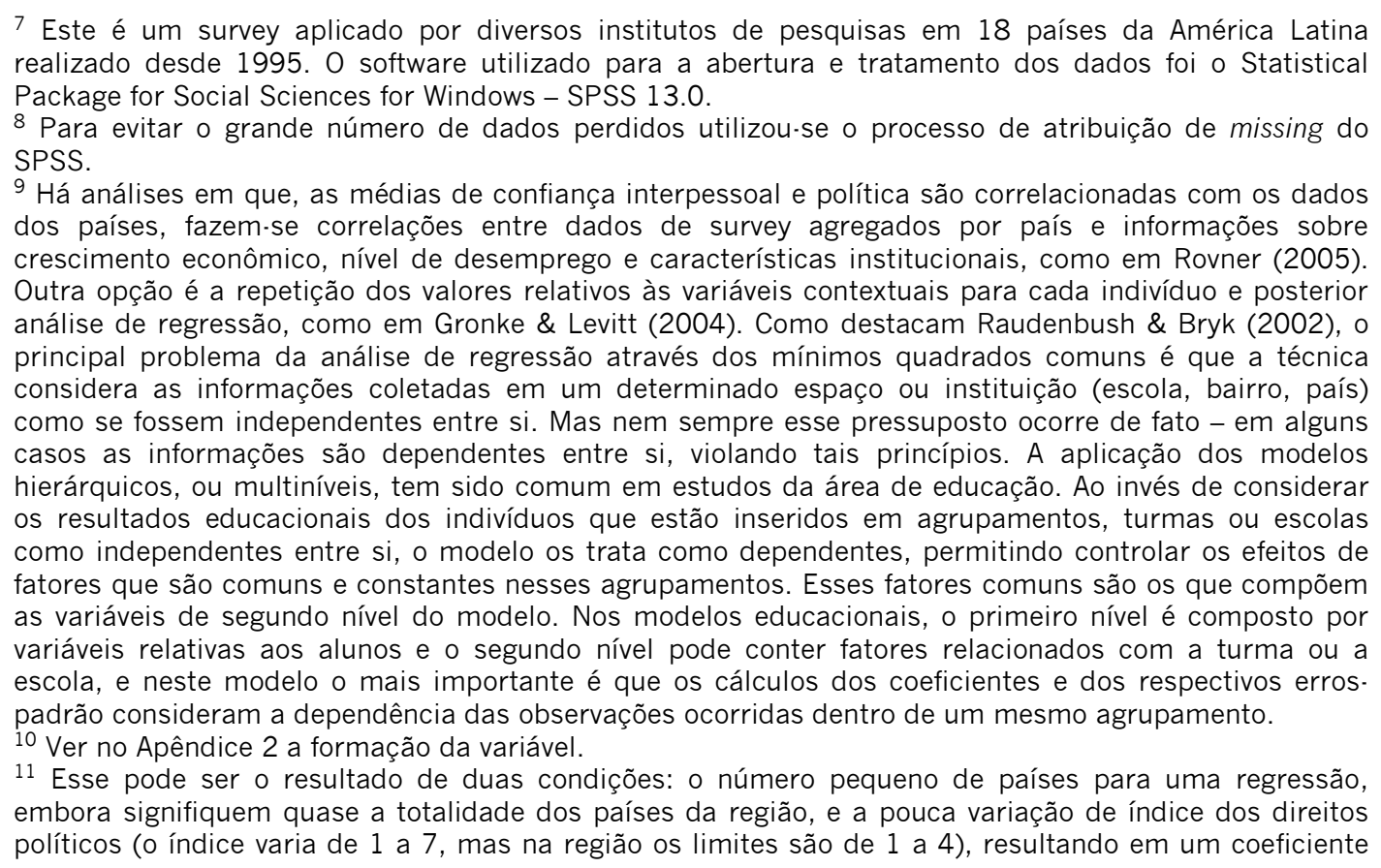


deste artigo, nível de democracia agregado por país, na percepção dos respondentes, será a proxi do nível de democracia do país. Considerando que o objetivo é aumentar a compreensão das covariantes da confiança nas instituições democráticas, essa percepção agregada é uma medida adequada para comparar países com níveis médios de democracia semelhantes ${ }^{12}$.

A estratégia de análise segue os padrões da análise hierárquica. O primeiro modelo é o mais simples (modelo nulo), o qual permite separar a variância não explicada, que é devida ao primeiro nível, daquela que é devida ao segundo nível. Em um momento posterior, são incluídas apenas as variáveis de segundo nível (médias como resultados). O terceiro modelo avalia o impacto do apoio ao presidente, controlado pelas variáveis sociodemográficas. Na sequência (modelo 4), são introduzidas as variáveis relativas às abordagens culturalistas e, no modelo final (modelo 5), são introduzidas as variáveis cognitivas e de avaliação de políticas públicas.

Ao contrário do método regressão linear (mínimos quadrados comuns) em que se utiliza o $\mathrm{R}^{2}$ como medida de ajuste do modelo escolhido, no método hierárquico, a avaliação do poder preditivo das variáveis independentes é decorrência da diminuição nos termos aleatórios: quanto maior a diminuição da variância não explicada, expressa pelos parâmetros $\sigma^{2}$ e $\tau_{00}$, maior poder preditivo têm as variáveis.

Para identificar as covariantes da confiança nas instituições na América Latina, este artigo testa quatro hipóteses: $1^{\text {a }}$ hipótese: o apoio ao presidente aumenta a confiança nas instituições políticas democráticas; $2^{a}$ hipótese: variáveis culturais e institucionais importam para confiança política, mas as últimas têm maior peso na explicação dessa; $3^{a}$ hipótese: parte do apoio ao presidente pode ser explicada pela satisfação das pessoas com a situação econômica e com o desempenho do governo; $4^{a}$ hipótese: em países com média de democracia percebida mais alta, a confiança é mais alta.

\section{Primeiro modelo: modelo nulo ou One-way-Anova}

A variável resposta $\mathrm{Y}_{\mathrm{ij}}$ é a confiança do indivíduo i no país j. Ela é será o resultado da equação que envolve a média de confiança nas instituições da população $\left(\gamma_{00}\right)$, o termo de erro relativo à variância entre os indivíduos $\left(r_{\mathrm{ij}}\right)$ e o

não significativo estatisticamente. Uma análise envolvendo um número maior de países pode permitir avaliar a força dessa variável.

12 Outras variáveis de segundo nível foram elaboradas, mas não se mostraram significativas para diferenciar os países. A agregação por país da variável que media o grau de percepção de que o Estado faz cumprir a lei era significativa sem a presença da que media o grau de democracia; como as variáveis têm alta correlação optou-se pela segunda, considerando seu efeito mais forte. 
termo de erro que é relativo às diferenças entre os países, as unidades de nível 2 $\left(u_{0} \mathrm{j}\right)$. A equação é a seguinte:

$$
\begin{gathered}
Y_{i j}=\beta_{0 j}+r_{i j} \\
\beta_{0 j}=\gamma_{00}+u_{0 j}
\end{gathered}
$$

Tabela 1

Confiança nas instituições

Modelo Nulo

\begin{tabular}{|c|c|c|c|c|}
\hline \multicolumn{1}{|c|}{ Efeito Fixo } & Coeficiente & Erro-padrão & $\mathbf{t}$ & Valor-p \\
\hline Média de confiança - Intercepto, $\mathrm{Y}_{00}$ & 39,385 & 1,898 & 20,751 & 0,000 \\
\hline Efeito Aleatório & Variância & Erro-padrão & Wald Z & Valor-p \\
\hline Variância entre os indivíduos, $\mathrm{r}_{\mathrm{ij}}\left(\sigma^{2}\right)$ & 547,179 & 5,444 & 100,509 & 0,000 \\
\hline Variância entre os países, $\mathrm{u}_{0 \text { i }}\left(\mathrm{T}_{00}\right)$ & 64,354 & 22,240 & 2,894 & 0,004 \\
\hline
\end{tabular}

Fonte: Latinobarômetro, 2005 N.20.222

A confiança média nas instituições é 39,38 pontos em uma escala que varia de 0 a 100 (ver no Apêndice a formação do índice). Como o valor do $\left(u_{0 j}\right)$ é significativo, rejeita-se a hipótese nula de que não há diferença na média de confiança nas instituições dos países. 0 percentual de variância devido aos países ${ }^{13}$ é de 10,52\% da variância total, o que justifica buscar as suas razões dessa.

\section{Segundo modelo: médias como resultados}

A questão a ser respondida agora é o quanto a média de confiança de cada país é influenciada por determinada característica desse nível de análise. O modelo inclui o grau médio de democracia percebida, a média de corrupção percebida e o nível de direitos políticos pela Freedom House. A equação é a seguinte:

$$
\begin{gathered}
\mathrm{Y}_{\mathrm{ij}}=\beta_{0 \mathrm{j}}+r_{1 \mathrm{j}} \\
\beta_{0 \mathrm{j}}=\gamma_{00}+\gamma_{01}(\text { corrupção })+\gamma_{02}(\text { direitos políticos })+\gamma_{02}(\text { percepção da corrupção })+u_{0 \mathrm{j}}
\end{gathered}
$$

13 Cálculo feito a partir da fórmula: $\tau_{00} /\left(\tau_{00}+\sigma_{2}\right)=64,354 /(64,354+547,179)=0,1052$ 
Tabela 2

Confiança nas instituições

\begin{tabular}{|c|c|c|c|c|}
\hline Efeito Fixo & Coeficiente & $\begin{array}{c}\text { Erro- } \\
\text { padrão }\end{array}$ & $\mathbf{t}$ & Valor-p \\
\hline $\begin{array}{c}\text { Média de confiança - Intercepto, } \\
\text { Yoo }\end{array}$ & 39,476 & 0,980 & 40,287 & 0,000 \\
\hline $\begin{array}{c}\text { Grau de democracia percebida } \\
\text { y01 }\end{array}$ & 5,430 & 1,436 & 3,780 & 0,002 \\
\hline $\begin{array}{c}\text { Direitos políticos (Freedom } \\
\text { House) y02 }\end{array}$ & 0,854 & 1,231 & 0,694 & 0,499 \\
\hline Corrupção percebida Y03 & $-0,331$ & 0,168 & $-1,969$ & 0,069 \\
\hline Efeito Aleatório & Variância & $\begin{array}{c}\text { Erro- } \\
\text { padrão }\end{array}$ & Wald Z & Valor-p \\
\hline $\begin{array}{c}\text { Variância entre os indivíduos, } \mathrm{r}_{\mathrm{ij}} \\
\left(\sigma^{2}\right)\end{array}$ & 547,179 & 5,444 & 100,509 & 0,000 \\
\hline Variância entre os países, $\mathrm{u}_{\mathrm{0j}}\left(\mathrm{T}_{00}\right)$ & 16,782 & 6,531 & 2,570 & 0,010 \\
\hline
\end{tabular}

Fonte: Latinobarômetro 2005 n. 20.222

Como se vê no modelo 2 da tabela 2, quanto mais democráticos os países na opinião dos indivíduos, maior a confiança nas instituições: um ponto a mais na média de democracia percebida aumenta em 5,43 pontos a confiança média nas instituições. O aumento de 10 pontos na percepção da corrupção diminuiu 3,31 pontos a confiança média nas instituições. $O$ índice de direitos políticos não foi significante estatisticamente, e está mantido nos modelos posteriores para efeito informativo. A introdução das duas variáveis levou a uma diminuição significativa de $73,92 \%$ da variância não explicada entre os países em torno das médias ${ }^{14}$.

\section{Terceiro modelo: apoio ao presidente com controles}

Neste modelo o objetivo é avaliar o efeito da variável "apoio ao presidente", mantidos os controles das variáveis sociodemográficas. E como é mais simples serve de linha de base para comparar com modelos que testam o efeito de variáveis consideradas importantes pelas teorias institucionalistas e culturalistas. A equação é a seguinte:

14 Proporção da Variância Explicada após introdução da variável Grau Médio de democracia percebida em $\beta_{0 j}:=\tau_{00}($ ANOVA $) \cdot \tau_{00}($ gdgmed $) / \tau_{00}($ ANOVA $)=(64,354 \cdot 116,782) / 64,354=0,7392$ 
$Y_{i j}=\beta_{0 j}+\beta_{1 j}(\operatorname{Seg} 26 a 35)+\beta_{2 j}(\operatorname{Seg} 26 a 35)+\beta_{3 j}\left(\right.$ Ter35a45) $+\beta_{4 j}($ Qua46a55 $)+$

$\beta_{5 \mathrm{j}}($ sexo $)+\beta_{6 \mathrm{j}}$ (fundamental completo) $+\beta_{7 \mathrm{j}}$ (médio completo) +

$\beta_{8 \mathrm{j}}$ (superior completo $)+\beta_{9 \mathrm{j}}$ (Nível socioeconômico $)+\beta_{10 \mathrm{j}}$ (Apoio ao presidente) $+r_{\mathrm{ij}}$

$\beta_{0 \mathrm{j}}=\gamma_{00}+\gamma_{01}$ (Corrupção) $+\gamma_{02}$ (Direitos políticos $)+\gamma_{02}$ (Percepção da corrupção $)+u_{0 \mathrm{j}}$

$\beta_{1 \mathrm{j} \ldots} \beta_{10 \mathrm{j}}=\gamma_{10} \ldots \gamma_{100}$

Tabela 3

Confiança nas instituições

\begin{tabular}{|c|c|c|c|c|}
\hline Efeito Fixo & Coeficiente & Erro-padrão & $\mathbf{t}$ & Sig. \\
\hline Média de confiança - Intercepto, y00 & 33,285 & 1,107 & 30,070 & 0,000 \\
\hline Grau de democracia percebida y01 & 3,944 & 1,392 & 2,834 & 0,013 \\
\hline Direitos políticos (Freedom House) y02 & 0,210 & 1,194 & 0,176 & 0,863 \\
\hline Corrupção percebida Y03 & $.0,128$ & 0,163 & $-0,786$ & 0,445 \\
\hline De 26 a 35 anos, y10 & $-2,011$ & 0,449 & $.4,479$ & 0,000 \\
\hline De 36 a 45 anos, y20 & $-2,564$ & 0,474 & $.5,407$ & 0,000 \\
\hline De 46 a 55 anos, y30 & $-3,096$ & 0,538 & $-5,753$ & 0,000 \\
\hline Acima de 56 anos, y40 & $-3,648$ & 0,506 & $.7,213$ & 0,000 \\
\hline sexo, y50 & 1,439 & 0,309 & 4,654 & 0,000 \\
\hline Fundamental completo y60 & 0,553 & 0,417 & 1,328 & 0,184 \\
\hline Médio completo y 70 & 0,606 & 0,492 & 1,232 & 0,218 \\
\hline Superior completo y80 & 2,213 & 0,748 & 2,957 & 0,003 \\
\hline Nível socioeconômico y90 & 0,349 & 0,202 & 1,723 & 0,085 \\
\hline Apoio ao presidente, $\mathrm{y} 100$ & 18,184 & 0,351 & 51,848 & 0,000 \\
\hline Efeito Aleatório & Variância & Erro-padrão & Wald Z & Valor-p \\
\hline Variância entre os indivíduos, $r_{\mathrm{ij}}\left(\sigma^{2}\right)$ & 480,480 & 4,782 & 100,484 & 0,000 \\
\hline Variância entre os países, $\mathrm{U}_{0 \mathrm{j}}\left(\mathrm{T}_{00}\right)$ & 15,772 & 6,130 & 2,573 & 0,010 \\
\hline
\end{tabular}

Fonte Latinobarômetro 2005 n. 20.222

Mantendo o controle das variáveis sociodemográficas, o apoio ao presidente aumenta em 18,18 pontos a confiança média. A introdução da variável representou uma diminuição de $12,18 \%$ da variância não explicada dentro dos países em comparação ao modelo anterior, indicando que esse é um importante fator da confiança política. Confirmando os achados da literatura em, Norris (1999a) e Bowler \& Donovan (2003), a confiança nas instituições tende a ser maior entre os "ganhadores", nesse caso, os apoiadores do presidente. O modelo evidenciou ainda que a confiança é maior entre os indivíduos com maior escolaridade e entre os homens, e menor entre os mais velhos.

É interessante notar que a introdução de "apoio ao presidente" levou a variável "percepção da corrupção" a perder a significância estatística. A 
identificação com o partido no poder, aqui como apoio ao presidente, não apenas melhora a avaliação das políticas do governo como também altera a percepção da corrupção no serviço público.

\section{Quarto modelo: testando o impacto das variáveis culturais}

Esse modelo tem o objetivo de testar o efeito do conjunto de variáveis culturais ${ }^{15}$. Teorias culturalistas defendem que essas variáveis deveriam ter impacto sobre a confiança porque elas indicariam os efeitos da socialização dos indivíduos sobre suas atitudes políticas. Para Catteberg \& Moreno (2005), a que a confiança interpessoal só não apresentou significância nas democracias estabelecidas - nos demais países, os que confiam nas outras pessoas também confiam mais nas instituições. Na América Latina, o argumento reforça a avaliação de uma "síndrome de desconfiança generalizada", mas vale destacar que vários autores, como Seligson (2002), defendem que são os anos continuados de democracia que levam ao aumento da confiança interpessoal, não o contrário ${ }^{16}$.

A satisfação com a vida também é variável tradicional dos estudos culturalistas. Como Inglehart (1997), defendemos que os indivíduos mais satisfeitos teriam mais confiança nas instituições. As outras três variáveis visam captar o quanto o indivíduo considera seus concidadãos cívicos: quanto mais cívicos forem considerados, espera-se que os indivíduos confiem mais nas instituições. Teriam essas variáveis importância significativa para reduzir a variância não explicada nos níveis de confiança política entre os indivíduos? Essa importância será medida pela diminuição que elas podem provocar na variância não explicada no nível individual, $\sigma^{2}$, em relação ao modelo anterior. A equação relativa ao modelo é a seguinte:

$Y_{i j}=\beta_{0 j}+\beta_{1 j}\left(\right.$ Seg26a35) $+\beta_{2 j}($ Seg26a35 $)+\beta_{3 j}\left(\right.$ Ter35a45) $+\beta_{4 j}($ Qua46a55 $)+$ $\beta_{5 \mathrm{j}}$ (sexo) $+\beta_{6 \mathrm{j}}$ (fundamental completo $)+\beta_{7 \mathrm{j}}$ (médio completo $)+$ $\beta_{8 \mathrm{j}}$ (superior completo $)+\beta_{9 \mathrm{j}}$ (Nível socioeconômico $)+\beta_{10 \mathrm{j}}$ (Apoio ao presidente) + $\beta_{11 \mathrm{j}}$ (Confiança interpessoal) $+\beta_{12 \mathrm{j}}$ (Satisfação com a vida) $+\beta_{13 \mathrm{j}}$ (Cumpridores da lei) $+\beta_{14 \mathrm{j}}$ (Exigem seus direitos) $+\beta_{15 \mathrm{j}}$ (Cientes das obrigações $)+r_{\mathrm{ij}}$

$\beta_{0 \mathrm{j}}=\gamma_{00}+\gamma_{01}$ (Corrupção) $+\gamma_{02}$ (Direitos políticos $)+\gamma_{02}($ Percepção da corrupção $)+u_{0 \mathrm{j}}$ $\beta_{1 \mathrm{j} \ldots} \beta_{10 \mathrm{j}}=\gamma_{10} \ldots \gamma_{150}$

15 Verificar no Apêndice 1 a descrição das variáveis e as estatísticas descritivas das mesmas.

16 O uso desse item tem sido muito criticado, como em Rennó \& Henrique (2008). Mas é a variável tradicionalmente usada e é a única disponível no Latinobarômetro 2005. 
Como se vê na Tabela 4, todas as variáveis foram significativas e com sinal na direção esperada, com destaque para a que indica que "os concidadãos são cumpridores da lei", - em que a percepção aumenta em 6,82 pontos a confiança nas instituições. Mas a contribuição do conjunto das variáveis não é muito significativa: elas diminuem a variância não explicada, $\sigma^{2}$, em apenas $3,71 \%$.

Tabela 4

Confiança nas instituições (Variáveis culturais - Modelo 4)

\begin{tabular}{|c|c|c|c|c|}
\hline Efeito Fixo & Coeficiente & Erro-padrão & $t$ & Sig. \\
\hline Média de confiança - Intercepto, y00 & 27,611 & 1,183 & 23,333 & 0,000 \\
\hline Grau de democracia percebida y01 & 2,950 & 1,474 & 2,001 & 0,065 \\
\hline Direitos políticos (Fredom House) y02 & 0,312 & 1,264 & 0,247 & 0,809 \\
\hline Corrupção percebida Y03 & $.0,126$ & 0,173 & $.0,729$ & 0,478 \\
\hline De 26 a 35 anos, $y 10$ & $-1,935$ & 0,441 & $.4,391$ & 0,000 \\
\hline De 36 a 45 anos, y20 & $.2,371$ & 0,466 & $.5,093$ & 0,000 \\
\hline De 46 a 55 anos, y30 & $-2,891$ & 0,529 & $.5,467$ & 0,000 \\
\hline Acimca de 56 anos, y40 & $.3,632$ & 0,497 & $.7,308$ & 0,000 \\
\hline sexo, y50 & 1,173 & 0,304 & 3,865 & 0,000 \\
\hline Fundamental completo y60 & 0,789 & 0,409 & 1,930 & 0,054 \\
\hline Médio completo y70 & 0,812 & 0,483 & 1,682 & 0,093 \\
\hline Superior completo y 80 & 2,214 & 0,735 & 3,011 & 0,003 \\
\hline Nível socioeconômico y90 & 0,414 & 0,200 & 2,075 & 0,038 \\
\hline Apoio ao presidente, $\mathrm{\gamma} 100$ & 16,782 & 0,348 & 48,212 & 0,000 \\
\hline Confiança interpessoal, y110 & 3,333 & 0,389 & 8,561 & 0,000 \\
\hline Satisfação com a vida, y120 & 2,168 & 0,346 & 6,261 & 0,000 \\
\hline Concidadãos cumpridores da lei, y130 & 6,828 & 0,413 & 16,549 & 0,000 \\
\hline Concidadãos exigem direitos, y140 & 2,557 & 0,323 & 7,915 & 0,000 \\
\hline Concidadãos cientes obrigações, y150 & 2,907 & 0,349 & 8,329 & 0,000 \\
\hline $\begin{array}{c}\text { Efeito Aleatório } \\
\end{array}$ & Variância & Erro-padrão & Wald Z & Valor-p \\
\hline Variância entre os indivíduos, $r_{i j}\left(\sigma^{2}\right)$ & 462,662 & 4,605 & 100,471 & 0,000 \\
\hline Variância entre os países, $\mathrm{U}_{0 j}\left(\mathrm{~T}_{00}\right)$ & 17,745 & 6,871 & 2,583 & 0,010 \\
\hline
\end{tabular}

Fonte Latinobarômetro 2005 n. 20.222 


\section{Modelo final: incluindo variáveis cognitivas e de avaliação das políticas}

Finalmente no quinto modelo, o objetivo é avaliar o impacto comparativo das variáveis típicas da teoria institucionalista ${ }^{17}$. São testadas as hipóteses relacionadas anteriormente, buscando verificar principalmente se parte do efeito do apoio ao presidente pode estar relacionada com a aprovação das políticas públicas e a satisfação com a situação econômica. Algumas pesquisas têm evidenciado que a percepção do indivíduo em relação à situação econômica, própria e do país, tem maior impacto sobre a confiança nas instituições do que medidas diretas de crescimento econômico (ver MCallister, 1999). Espera-se que os mais satisfeitos confiem mais.

Outra variável importante para entender a confiança nas instituições é o interesse por política. Criamos um índice de sofisticação ${ }^{18}$ com o objetivo de avaliar se a atenção e interesse em relação aos assuntos políticos aumenta a confiança. Como a variável é influenciada pela escolaridade, assim como pela experiência política na vida adulta, ela pode captar o efeito da experiência política pós. socialização. Nesse caso espera-se que aqueles com maior sofisticação, mais inseridos nas regras do jogo político, apresentem maior confiança.

A introdução das variáveis institucionalistas - avaliação da situação econômica, das políticas públicas e a percepção do combate à corrupção - visa operacionalizar a satisfação com o funcionamento das instituições. O objetivo é distinguir entre o apoio puramente afetivo ao presidente, o que qualificaria os ganhadores, e a transferência de apoio em função da satisfação com políticas públicas. A hipótese é que os indivíduos mais satisfeitos com serviços de saúde e educação têm maior confiança política. Quanto à percepção do combate à corrupção, espera-se que aqueles que acreditam que a corrupção esteja sendo combatida confiem mais nas instituições. Para alguns autores, como Catterberg \& Moreno (2005), a América Latina existiria uma "tolerância" em relação à corrupção, mas no seu estudo essa variável não se mostrou significativa na região.

A equação relativa ao quinto modelo (Tabela 5) é a seguinte ${ }^{19}$ :

$Y_{i j}=\beta_{0 j}+\beta_{1 j}\left(\right.$ Seg26a35) $+\beta_{2 j}\left(\right.$ Seg26a35) $+\beta_{3 j}\left(\right.$ Ter35a45) $+\beta_{4 j}\left(\right.$ Qua46a55) $+\beta_{5 j}$ (sexo) $+\beta_{6 \mathrm{j}}$ (fundamental completo) $+\beta_{7 \mathrm{j}}$ (médio completo) $+\beta_{8 \mathrm{j}}$ (superior completo) $+\beta_{9 \mathrm{j}}$ (Nível socioeconômico) $+\beta_{10 \mathrm{j}}$ (Apoio ao presidente) $+\beta_{11 \mathrm{j}}$ (Confiança interpessoal $)+\beta_{12 j}$ (Satisfação com a vida $)+\beta_{13 j}$ (Cumpridores da lei $)+\beta_{14 j}($ Exigem

\footnotetext{
17 Verificar no Apêndice 1 a descrição das variáveis e as estatísticas descritivas das mesmas.

18 A composição do nível de sofisticação política é orientada pelo trabalho de Reis \& Machado (2001). Como as variáveis de conhecimento dos assuntos políticos não estão disponíveis no Latinobarômetro, a proxi utilizada foi a declaração do entrevistado sobre o próprio nível de conhecimento.

${ }^{19}$ A descrição do modelo segue o anterior com as novas variáveis. Efeitos aleatórios não foram incluídos.
} 
seus direitos $)+\beta_{15 \mathrm{j}}$ (Cientes das obrigações $)+\beta_{16 \mathrm{j}}$ (Nível satisfação econômica) + $\beta_{17 j}$ (Nível de sofisticação) $+\beta_{18 j}$ (Políticas públicas iguais) $+\beta_{19 j}$ (Políticas públicas melhores $)+\beta_{20 \mathrm{j}}$ (Algum e muito combate à corrupção $)+r_{\mathrm{ij}}$

Tabela 5

Confiança nas instituições (Modelo 5 - completo)

\begin{tabular}{|c|c|c|c|c|}
\hline Efeito Fixo & Coef. & Erro-padrão & $\mathbf{t}$ & Sig. \\
\hline Média de confiança - Intercepto, y00 & 11,278 & 1,230 & 9,170 & 0,000 \\
\hline Grau de democracia percebida y01 & 2,157 & 1,406 & 1,534 & 0,147 \\
\hline Direitos políticos (Freedom House) y02 & 0,091 & 1,205 & 0,076 & 0,941 \\
\hline Corrupção percebida Y03 & $-0,117$ & 0,165 & $-0,709$ & 0,490 \\
\hline De 26 a 35 anos, y 10 & $-1,381$ & 0,425 & $-3,247$ & 0,001 \\
\hline De 36 a 45 anos, y20 & $-1,152$ & 0,451 & $-2,553$ & 0,011 \\
\hline De 46 a 55 anos, $\mathrm{y} 30$ & $\cdot 1,477$ & 0,513 & $-2,879$ & 0,004 \\
\hline Acima de 56 anos, y40 & $-1,590$ & 0,484 & $-3,283$ & 0,001 \\
\hline sexo, y50 & 0,192 & 0,295 & 0,652 & 0,515 \\
\hline Fundamental completo y60 & 0,390 & 0,395 & 0,989 & 0,322 \\
\hline Médio completo y70 & $.0,231$ & 0,469 & $-0,492$ & 0,623 \\
\hline Superior completo y 80 & 0,456 & 0,716 & 0,636 & 0,525 \\
\hline Nível socioeconômico y90 & $.0,138$ & 0,194 & $.0,712$ & 0,477 \\
\hline Apoio ao presidente, y100 & 13,006 & 0,350 & 37,169 & 0,000 \\
\hline Confiança interpessoal, y110 & 2,325 & 0,376 & 6,182 & 0,000 \\
\hline Satisfação com a vida, y120 & 0,324 & 0,341 & 0,949 & 0,343 \\
\hline Concidadãos cumpridores da lei, y130 & 5,162 & 0,400 & 12,907 & 0,000 \\
\hline Concidadãos exigem direitos, y140 & 1,862 & 0,312 & 5,970 & 0,000 \\
\hline Concidadãos cientes obrigações, y150 & 2,489 & 0,337 & 7,389 & 0,000 \\
\hline Nível avaliação situação econômica, y160 & 3,828 & 0,194 & 19,725 & 0,000 \\
\hline Nível sofisticação, y170 & 4,325 & 0,232 & 18,674 & 0,000 \\
\hline Políticas públicas iguais, y180 $\left(^{* * *}\right)$ & 3,023 & 0,375 & 8,070 & 0,000 \\
\hline Políticas públicas melhores, y190 & 6,648 & 0,375 & 17,724 & 0,000 \\
\hline Algum e muito combate à corrupção, y200 & 4,611 & 0,327 & 14,095 & 0,000 \\
\hline Efeito Aleatório & Variância & Erro-padrão & Wald Z & $\begin{array}{c}\text { Valor } \\
\mathbf{p}\end{array}$ \\
\hline Variância entre os indivíduos, $r_{i j}\left(\sigma^{2}\right)$ & 429,711 & 4,277 & 100,459 & 0,000 \\
\hline Variância entre os países, $\mathrm{U}_{0 \mathrm{j}}\left(\mathrm{T}_{00}\right)$ & 16,132 & 6,251 & 2,581 & 0,010 \\
\hline
\end{tabular}

Fonte Latinobarômetro 2005 n. 20.222 
Todos os coeficientes das variáveis introduzidas no modelo foram positivos e significativos, valendo destacar: o aumento em um nível da satisfação econômica aumenta a confiança, em média, em 3,83 pontos; o aumento em um nível da sofisticação política aumenta, em média, a confiança em 4,33 pontos; a percepção de que há algum e muito combate à corrupção aumenta, em média, a confiança em 4,61; achar que as políticas estão iguais - comparando com os que acham que as políticas públicas estão piores aumenta em 3,02 pontos a confiança, e achar que as políticas estão melhores aumenta em 6,65 pontos a confiança nas instituições.

O efeito médio do "apoio ao presidente" sobre a confiança nas instituições entre os países $\left(\gamma_{10}\right)$ foi reduzido com a introdução de variáveis de avaliação do regime, passando de 16,78, no modelo anterior, para 13,00. Essa alteração evidencia que parte do efeito do "apoio ao presidente" era devida às variáveis de avaliação das políticas que foram introduzidas no modelo. É interessante notar que a variável "grau médio de democracia percebida" perde a significação estatística, indicando que uma parte do efeito do "grau de democracia" no país é explicada pelas variáveis de avaliação de desempenho do regime. O modelo final, com a inclusão dessas variáveis, reduziu em $7,15 \%$ a variância entre os indivíduos, em relação ao modelo anterior. Um resultado muito superior do que aquele alcançado pelas variáveis culturais.

Com a introdução das variáveis cognitivas e de avaliação perdem significância estatística as variáveis escolaridade, sexo, nível socioeconômico e satisfação com a vida. Vale destacar que a introdução da avaliação da situação econômica fez com que a satisfação com a vida e o nível socioeconômico perdessem significação. Essa pode ser uma evidência de que a percepção da situação econômica seja mais importante do que a posição econômica objetiva do indivíduo. Embora os teóricos culturalistas insistam em manter a variável "satisfação com a vida" em pesquisas sobre confiança política, o resultado aqui encontrado permite sugerir que a percepção da satisfação econômica é uma medida mais adequada para predizer a confiança política.

O comportamento geral das variáveis corresponde às expectativas iniciais, ou seja, tanto os fatores relacionados com a avaliação de desempenho quanto a cultura política demonstraram ser importantes para predizer a confiança política. Os indivíduos que avaliam positivamente os resultados da democracia confiam mais nas instituições do regime. Da mesma forma, a confiança pessoal e a avaliação dos concidadãos têm um coeficiente positivo, indicando que os que confiam mais nos outros e consideram os concidadãos como cívicos têm níveis mais altos de confiança política. O modelo final contribuiu de modo importante para a definição das principais covariantes da confiança política na região: permitiu uma redução de $21,47 \%$ da variância não explicada da confiança nas instituições dentro dos países $\left(\sigma^{2}\right)$, em relação ao modelo nulo, e uma redução de $74,9 \%$ na variância não 
explicada entre os países $\left(\tau_{00}\right)$. Mas cabe destacar que as variáveis relativas às experiências dos cidadãos com o regime na vida adulta, o apoio ao presidente e a avaliação das políticas, foram responsáveis por $90 \%$ da redução na variância no interior dos países.

Em um modelo não apresentado verificou-se que o coeficiente aleatório relativo à confiança pessoal é significativo, indicando que o efeito da confiança interpessoal sobre a confiança nas instituições varia entre países. Para citar um exemplo dessa diversidade, uma regressão linear de dois países com melhores classificações de democracia e com maiores níveis de confiança nas instituições, Costa Rica e Uruguai, indica resultados opostos. A confiança interpessoal não alcança significância estatística para explicar a confiança política no primeiro país e no segundo tem sinal negativo, indicando que aqueles que confiam nas outras pessoas tem menor confiança nas instituições. A hipótese culturalista de que a confiança interpessoal aumenta a confiança nas instituições é parcialmente corroborada, mas pesquisas posteriores devem explicar por que o seu impacto é diferente entre os países. Outra hipótese pode ser aquela sustentada por Rennó \& Castro (2008), de que é necessário utilizar questões mais apropriadas para avaliar a confiança interpessoal.

\section{Considerações finais}

A análise realizada neste artigo corroborou, principalmente, as hipóteses elaboradas a partir da literatura institucionalista. Nos países da América Latina, a avaliação do desempenho das instituições é um fator-chave para o entendimento da confiança política, tanto pelo bem-estar econômico e políticas públicas que promovem quanto pelo combate à corrupção percebido pelos indivíduos. Além disso, a sofisticação política apresentou um desempenho mais importante do que a educação formal. Essas são evidências de que a experiência com o regime democrático é mais importante do que o aprendizado ocorrido durante a socialização primária para explicar a confiança política. Outra conclusão é de que as médias de confiança nas instituições são maiores nos países considerados mais democráticos, o que mostra que os indivíduos levam em conta a qualidade da democracia para avaliar as instituições.

Esses resultados reforçam as conclusões de que os níveis de confiança nas instituições democráticas são baixos na região. Mas é necessário lembrar que o próprio regime democrático tem pouco tempo de vigência nesses países, os quais passaram por experiências autoritárias nos anos setenta. É possível entender essa realidade como fizeram Mattes e Bratton (2007) em uma análise sobre a África: o efeito combinado do aumento da atenção em relação às questões políticas, das 
avaliações de desempenho e da experiência direta com a competição política pode permitir um aprendizado em relação à dinâmica do regime.

Uma análise parcial desse fenômeno poderia levar à conclusão de que haveria na região a preponderância do populismo decorrente de aspectos carismáticos da figura do presidente. No entanto, a análise permitiu localizar um componente racional do aumento da confiança política decorrente do apoio ao presidente: o indivíduo avalia se as instituições estão produzindo efeitos positivos sobre o seu bem-estar. A explicação mais adequada é que aqueles identificados como "ganhadores" no processo eleitoral, os que apóiam o presidente, têm maior confiança nas instituições do que os "perdedores", os que não apóiam o presidente. A importância do efeito do apoio ao presidente também pode ser uma evidência de que os latinoamericanos, assim como qualquer cidadão de novas democracias, transferem as avaliações que fazem das autoridades políticas que estão no poder para as instituições do regime, como indicou McAlister (1999). Mas, como foi evidenciado pela análise, parte do efeito desse apoio foi explicada pela avaliação que os indivíduos fazem do desempenho das instituições, avaliadas pelo bem-estar econômico, pelas políticas produzidas e pela capacidade de combater a corrupção.

Os resultados aqui obtidos sugerem que é possível melhorar os níveis de confiança na democracia se os governos forem capazes de promover políticas públicas de qualidade e combater a corrupção. Uma interpretação alternativa poderia ser a de que a confiança nas instituições está muito vinculada a fatores "instrumentais" e contextuais, os quais poderiam ser alterados em situações de crise. Mas vale destacar que esses resultados aproximam-se dos resultados das pesquisas feitas nas democracias estabelecidas e em países da África. Mais do que configurar um quadro distinto de outras regiões em função da, "síndrome de desconfiança generalizada", como alguns pesquisadores defendem, a América Latina possui covariantes da confiança política que podem ser entendidas à luz das teorias institucionalistas.

Os resultados permitem, por fim, uma ponderação do argumento de que o efeito do apoio ao presidente seria apenas outra face da herança cultural personalista e da identificação carismática, evidências da sobrevivência do populismo na região. Ao mesmo tempo em que a avaliação do presidente influencia a percepção que se tem das instituições, também são importantes as avaliações feitas dos resultados econômicos e políticos do regime democrático, traduzidas no aumento da confiança nas instituições.

\section{Referências Bibliográficas}

ALMOND, G.; VERBA, S. The Civic Culture: Political Attitudes and Democracy in Five Nations. Princeton: Princeton University Press, 1963. 
ANDERSON, C. J. Center on Democratic Performance Working, Paper, Binghamton University, n.102 [Online]. Political Satisfaction in Old and New Democracies, 2001. Disponível em: <http://cdp.binghamton.edu/papers.html>.

ANDERSON, C.J; GUILORY, C. A. "Political Institutions and satisfaction with Democracy: A cross-national analysis of consensus and majoritarian systems". The American Political Science Review, v.91, p.66-81, Mar. 1997.

BOWLER, S.; DONOVAN, T. The Effects of Winning and Losing on Attitudes about Political Institutions and Democracy in the United States. Chicago IL, 3.6, Midwest Political Science Association Meeting, Apr. 2003.

BRATTON, M.; MATTES, R. Support for democracy in Africa: Intrinsic or Instrumental? British Journal of Political Science, v.31, p.447-474. 2001.

.Wide But Shallow: Popular support for democracy in Africa. [Online] Afrobarometer Working Papers, n.19. 2002. Disponível em: <www.afrobarometer.org>.

CASTRO, M. M. M. Determinantes do Comportamento Eleitoral: A centralidade da Sofisticação Política. Tese de Doutorado, IUPERJ. 1994.

CATTEBerg, G; MORENO, A. "The Individual Bases of Political Trust: Trends in new and established democracies". International Journal of Public Opinion Research, v.18, n. 1, p.31-48, Aug. 2005.

CROZIER, M.; HUNTINGTON, S.; WATANUKI, J. The Crisis of Democracy: Report on the Governability of Democracies to the Trilateral Commission. In: PHARR, S. J.; PUTNAM, R. (eds.). Desaffected Democracies: What's Troubling the Trilateral Coutries? Princeton: Princeton University Press, p.3-27, 1975.

EASTON, D. A Systems Analysis of Political Life. New York: John Wiley and Sons Inc, 1965.

GRONKE, P.; LEVITT, B. Delegative Democracy and Confidence in Legislatures: Explaining institutional authority and trust in Latin America. Las Vegas. p.7-9, NV. Paper presented at the XXV Congress of the Latin American Studies Association, Sept. 2004.

HeTHERINGTON, M. J. "The Political Relevance of Political Trust". American Political Science Review, v.92, p.791-808. 1998.

HUNTINGTON, S. P. Foreword. In: PHARR, S. J.; PUTNAM, R. (eds.). Desaffected Democracies: What's Troubling the Trilateral Countries? Princeton: Princeton University Press, 2000. 
INGLEHART, R. Modernization and Postmodernization: Cultural, economic, and political change in 43 societies. Princeton: Princeton University Press, 1997.

KING, G.; HONAKER, J. JOSEPH, A.; SCHEVE, K. "Analysing incomplete data: An alternative algorithm for multiple imputation." American Political Science Review, 95(1), p.46-69, 2001.

LAGOS, M. "A Máscara Sorridente da América Latina." Opinião Pública, Campinas, vol.6, n.1, p.1-16, abril, 2000.

LATINOBARÔMETRO. Informe de Prensa, 2006.

LEVI, M.; STOKER, L. "Political Trust and Trustworthiness." Annual Review of Political Science, n.3, p.475-507, 2000.

LOPES, D. M. N. N. "Para Pensar a Confiança e a Cultura Política na América Latina." Opinião Pública, Campinas, vol. X, n.1, p.162-167, maio, 2004.

MAINWARING, S.; BRINKS, D.; PÉREZ-LIÑAN, A. "Classificando Regimes Políticos na América Latina, 1945-1999." Dados, Rio de Janeiro, v.44, n.4, p.645-687, 2001.

MATTES, R.; BRATTON, M. "Learning about Democracy in Africa: Awareness, Performance, and Experience." America Journal of Political Science, v.51, n.1, p.192. 217, Jan, 2007.

MCALLISTER, I. "The Economic Performance of Governments." In: NORRIS, P. (org). Critical Citizens: Global support for democratic government. Oxford: Oxford University Press, p.88-203, 1999.

MILLER, A.; LISHTAUG, O. "Political Performance e Institutional Trust." In: NORRIS, P. (org.) Critical Citizens: global support for democratic government. Oxford: Oxford University Press, p.204-216.

MISHLER, W.; ROSE, R. "What are the Origins of Political Trust? Testing Institutional and Cultural Theories in Post-Communist Societies." Comparative Political Studies, v.34, n.1, p.30-62, Feb. 2001.

MOISÉS, J. A. "A desconfiança nas instituições democráticas." Opinião Pública, v.11, n.1, Campinas, mar. 2005a.

. "Cidadania, confiança e instituições democráticas." Lua Nova, n.65, São Paulo, maio/ago. 2005b.

NORRIS, P. "Introduction: The Growth of Critical Citizens?" In: NORRIS, P. (org.). Critical Citizens: global support for democratic government. Oxford: Oxford University Press, p.1-27, 1999. 
Institutional Explanations for Political Support. In: NORRIS, P. (org.). Critical Citizens: global support for democratic government. Oxford: Oxford University Press, p.217-235. 1999a.

Conclusion: The Growth of Critical Citizens and its Consequences. In: NORRIS, P. (org.). Critical Citizens: global support for democratic government. Oxford: Oxford University Press, p.257-272, 1999b.

NORRIS, P.; NEWTON, K. Confidence in Public Institutions: Faith, Culture, or Performance? In: PHARR, S. J.; PUTNAN, R. (ed.). Desaffected Democracies: What's Troubling the Trilateral Countries? Princeton: Princeton University Press, p.52.73, 2000.

POWER, T. J.; JAMISON, G. D. "Desconfiança Política na América Latina.” Opinião Pública, Campinas, vol. XI, n. 1, p.64-93, mar. 2005.

PUTNAM, R. D.; PHARR, S. J.; DALTON, R. J. Introduction: What's Troubling the Trilateral Democracies? In: PHARR, S. J.; PUTNAN, R. (ed.). Desaffected Democracies: What's Troubling the Trilateral Countries? Princeton, Princeton University Press, p.3-27. 2000.

RAUDENBUSH, S. W.; BRYK, A. Hierarchical Linear Models. Applicattions and Data Analysis Methods, California: Sage Publications, 2002.

REIS, F. W.; CASTRO, M. M. M. "Democracia, Civismo e Cinismo: Um estudo empírico sobre normas e racionalidade." Revista Brasileira de Ciências Sociais, vol. 16, n.45, São Paulo, fevereiro, 2001.

RENNÓ, L.; CASTRO, H. Assessing the Validity and Reliability of Interpersonal Trust Measures [Online]. Cross-National Surveys, 2008. Disponível em:

<http://sitemason.vanderbilt.edu/files/fFbeBq/Renno_Measuring_Interpersonal_Tru st_revised\%5b1\%5d.pdf>.

ROSE, R.; SHIN, D. C.; MUNRO, N. "Tensions Between the Democratic Ideal and Reality: South Korea." In: NORRIS, P. (ed.). Critical Citizens: global support for democratic government. Oxford: Oxford University Press, p.146-168, 1999.

ROSE, R. "Perspectives on political behavior in time and space." In: DALTON, R. J.; KLINGEMANN, H. (eds.). Oxford Handbook of Political Behavior. Oxford: Oxford University Press, p.283-301. 2007.

ROVNER, H. Support for Democracy in Latin America in the 1990s: A comparative empirical inquiry. Tese de doutorado, Department of Government, University of Essex, 2005. 
SELIGSON, M. A. "The Renaissance of Political Culture or the Renaissance of the Ecological Fallacy?" Comparative Politics, Apr. 2002.

Apêndice 1

Medidas descritivas das variáveis independentes

\begin{tabular}{|c|c|c|}
\hline Variável & (N) & $(\%)$ \\
\hline \multicolumn{3}{|l|}{ Grupos etários } \\
\hline 1 até 25 anos & 5064 & 25,04 \\
\hline 2 de 26 a 35 anos & 4727 & 23,38 \\
\hline 3 de 36 a 45 anos & 4005 & 19,81 \\
\hline 4 de 46 a 55 anos & 2658 & 13,14 \\
\hline 556 anos ou mais & 3768 & 18,63 \\
\hline Total & 20222 & 100,00 \\
\hline \multicolumn{3}{|l|}{ Sexo } \\
\hline Mulher & 10296 & 50,91 \\
\hline Homem & 9926 & 49,09 \\
\hline Total & 2022 & 100,00 \\
\hline \multicolumn{3}{|l|}{ Escolaridade } \\
\hline $\begin{array}{l}\text { Até fundamental } \\
\text { incompleto }\end{array}$ & 6310 & 31,20 \\
\hline $\begin{array}{l}\text { Fundamental } \\
\text { completo }\end{array}$ & 7462 & 36,90 \\
\hline Médio completo & 1279 & 6,30 \\
\hline Superior completo & 1279 & 6,30 \\
\hline Total & 20222 & 68,80 \\
\hline \multicolumn{3}{|c|}{ Nível socioeconômico } \\
\hline Muito baixo & 5748 & 28,42 \\
\hline Baixo & 6985 & 34,54 \\
\hline Médio & 5882 & 29,09 \\
\hline Alto & 1607 & 7,95 \\
\hline Total & 20222 & 100,00 \\
\hline \multicolumn{3}{|l|}{ Apoio ao presidente } \\
\hline Não apóia & 13161 & 65,08 \\
\hline Apóia & 7061 & 34,92 \\
\hline Total & 20222 & 100,00 \\
\hline \multicolumn{3}{|c|}{ Confiança interpessoal } \\
\hline Não pode confiar & 16246 & 80,34 \\
\hline Pode confiar & 3976 & 19,66 \\
\hline Total & 20222 & 100,00 \\
\hline
\end{tabular}

\begin{tabular}{|l|c|c|}
\hline \multicolumn{3}{|l|}{ Satisfação com a vida } \\
\hline Não satisfeito & 6067 & 30,00 \\
\hline Satisfeito & 14155 & 70,00 \\
\hline Total & 20222 & 100,00 \\
\hline Concidadãos cumpridores da lei \\
\hline Não cumprem & 16126 & 79,74 \\
\hline Cumprem & 4096 & 20,26 \\
\hline Total & 20222 & 100,00 \\
\hline Concidadãos exigem
\end{tabular}

Concidadãos exigem seus direitos

\begin{tabular}{|l|c|c|}
\hline Não exigem & 9561 & 47,28 \\
\hline Exigem & 10661 & 52,72 \\
\hline Total & 20222 & 100,00 \\
\hline
\end{tabular}

Concidadãos conscientes obrigações

\begin{tabular}{|l|c|c|}
\hline Não & 13264 & 65,59 \\
\hline Sim & 6958 & 34,41 \\
\hline Total & 20222 & 100,00 \\
\hline
\end{tabular}

\section{Sofisticação política}

\begin{tabular}{|l|c|c|}
\hline Baixa & 10043 & 49,66 \\
\hline Média & 7878 & 38,96 \\
\hline Alta & 2301 & 11,38 \\
\hline Total & 20222 & 100,00 \\
\hline
\end{tabular}

Avaliação das políticas públicas

\begin{tabular}{|l|c|c|}
\hline Piorou & 5796 & 28,66 \\
\hline Igual & 9341 & 46,19 \\
\hline Melhorou & 5085 & 25,15 \\
\hline Total & 2022 & 100,00 \\
\hline
\end{tabular}

\section{Combate à corrupção}

\begin{tabular}{|l|c|c|}
\hline Nenhum e pouco & 13715 & 67,82 \\
\hline Algum e muito & 6507 & 32,18 \\
\hline Total & 20222 & 100,00 \\
\hline
\end{tabular}

Fonte: Latinobarômetro, 2005 
Estatísticas descritivas

Distribuição, por país, da confiança nas instituições e das variáveis de segundo nível

\begin{tabular}{|l|c|c|c|c|}
\hline \multicolumn{1}{|c|}{ País } & $\begin{array}{c}\text { Média confiança } \\
\text { nas instituições }\end{array}$ & $\begin{array}{c}\text { Grau médio de } \\
\text { democracia }\end{array}$ & $\begin{array}{c}\text { Direitos } \\
\text { políticos (*) }\end{array}$ & $\begin{array}{c}\text { Corrupção } \\
\text { média } \\
\text { percebida }\end{array}$ \\
\hline Argentina & 41,29 & 0,02 & $-0,34$ & 3,71 \\
\hline Bolívia & 33,90 & $-0,30$ & 0,66 & 4,48 \\
\hline Brasil & 40,98 & $-0,23$ & $-0,34$ & $-4,99$ \\
\hline Colômbia & 45,32 & 0,38 & 1,66 & $-2,32$ \\
\hline Costa Rica & 42,94 & 1,05 & $-1,34$ & $-3,96$ \\
\hline Chile & 45,82 & 0,77 & $-1,34$ & $-20,99$ \\
\hline Ecuador & 27,83 & $-1,03$ & 0,66 & 14,27 \\
\hline El Salvador & 36,48 & $-0,26$ & $-0,34$ & 0,91 \\
\hline Guatemala & 33,18 & $-0,79$ & 1,66 & 7,21 \\
\hline Honduras & 41,05 & $-0,26$ & 0,66 & 0,38 \\
\hline México & 40,83 & $-0,40$ & $-0,34$ & 9,00 \\
\hline Nicarágua & 24,45 & $-0,99$ & 0,66 & 7,14 \\
\hline Panamá & 31,87 & $-0,24$ & $-1,34$ & $-0,14$ \\
\hline Paraguai & 35,90 & $-1,46$ & 0,66 & 1,92 \\
\hline Peru & 34,93 & $-0,83$ & $-0,34$ & 5,35 \\
\hline Uruguai & 57,86 & 1,66 & $-1,34$ & $-19,53$ \\
\hline Venezuela & 50,17 & 2,08 & 0,66 & $-3,19$ \\
\hline Rep. & 44,11 & 0,67 & $-0,34$ & 3,22 \\
\hline Dominicana & & & & \\
\hline Fonte: & & & \\
\hline
\end{tabular}

Fonte: Latinobarômetro e (*) Freedom House N.18

\section{Apêndice 2}

1 Variável dependente

Índice de confiança nas instituições - Quanta confiança você tem? Judiciário, Congresso, Administração Pública e Partidos políticos. Recodificação: muita (3), alguma (2), pouca (1), não sabe e não respondeu (0), nenhuma confiança (-1).

Foi criado um fator único sem rotação com KMO 0,771. As cargas fatoriais foram normalizadas para um índice de 0-100.

2 Variáveis independentes

2.1 Variáveis de segundo nível

2.1.1 Grau de democracia percebida - "Com uma escala de 1 a 10 pedimos que você avalie quão democrático é o país (nome do país)". O (1) quer dizer não democrático e o (10) quer dizer que o país é totalmente democrático.

A variável foi agregada por país e centralizada pela média de todos os países. 
2.1.2 Direitos políticos Freedom House - Pontuação entre 1 a 7 , variando do mais democrático (1) até o menos democrático (7).

2.1.3 Corrupção percebida - "Imagine que o total dos funcionários seja 100 e que você teria de dizer quantos desses 100 você crê que sejam corruptos". [A variável foi agregada por país e centralizada pela média de todos os países.]

2.2 Variáveis de primeiro nível

2.2.1 Variáveis culturais

2.2.1.1 Confiança interpessoal - "Em geral, você diria que se pode confiar na maioria das pessoas ou que nunca se é suficientemente cuidadoso no trato com os demais?" Nunca se é suficientemente cuidadoso (0); é possível confiar na maioria das pessoas (1).

2.2.1.2 Satisfação com a vida - "Em termos gerais, você diria que está satisfeito com sua vida?" Nada satisfeito e não muito satisfeito (0); bastante satisfeito e muito satisfeito (1).

2.2.1.3 Avaliação dos compatriotas - foram usadas três variáveis: 1. "Você diria que os compatriotas (nacionalidade) cumprem as leis?": nem um pouco e um pouco (0), bastante e muito (1); 2 . São exigentes dos seus direitos: nem um pouco e um pouco (0), bastante e muito (1); são conscientes das suas obrigações: nem um pouco e um pouco (0), bastante e muito (1).

\subsubsection{Variáveis cognitivas e institucionais}

2.2.2.1 Apoio ao presidente - 1. "Você aprova ou desaprova a gestão do governo encabeçado pelo presidente (nome do presidente do país)?" a. Aprova (0), b. Não aprova (3); 2. Por favor, olhe essa relação e diga-me quanta confiança tem no presidente: muita (3), alguma (2), pouca (1), nenhuma (0). [Os pontos foram somados variando de 1 a 6 , assim recodificados: de 1 a 4 pontos como não aprova (0) e de 5 a 6 pontos como aprova (1).]

2.2.2.2 Nível de avaliação da situação econômica (individual e do país) - 1. "Como você avaliaria a situação econômica atual do país?" Diria que é: muito boa, boa, regular, ruim, muito ruim; 2. "Você considera que a situação econômica atual do país comparando com doze meses atrás: é muito melhor, um pouco melhor, igual, um pouco pior, ou muito pior?" 3. "Como você avaliaria a sua situação econômica e da sua família atual?" Diria que é: muito boa, boa, regular, ruim, muito ruim; 4. "Você considera que a sua situação econômica e da sua família atual comparando com doze meses atrás é muito melhor, um pouco melhor, igual, um pouco pior, ou muito pior". [Os pontos foram somados em um índice que varia de 4 a 20, recodificados em quatro níveis: de 4 a 8 como situação econômica muito ruim (1), 
de 9 a 11 como situação econômica ruim (2), de 12 a 14 como situação econômica boa (3), de 15 a 20 como situação econômica muito boa (4).]

2.2.2.3 Nível de sofisticação, elaborado a partir de três questões - 1. "Há pessoas que dizem que a política é tão complicada que com frequência não dá para entender o que se passa" (1), outros opinam que a política não é complicada e se pode entender o que se passa (2); 2. "Quanto você está interessado na política?" muito interessado (4); interessado (3); algum interesse em política (2); pouco interessado (1); nada interessado (0); 3. "Quanto conhecimento você diria que tem dos acontecimentos políticos e sociais no seu país?" Você diria que conhece: muito (3); conhece bastante (2); conhece pouco (1); não sabe nada ou quase nada (0). [Os pontos foram somados em um índice que varia de 1 a 10 , recodificados em três níveis: de 1 a 3 pontos como sofisticação baixa; de 4 a 6 pontos como média; de 7 a 10 como alta.]

2.2.2.4 Avaliação das políticas públicas, elaborada a partir de duas questões: "Qualidade dos Hospitais Públicos nos últimos 12 meses e Qualidade das Escolas Públicas nos últimos 12 meses" - piorou muito (1), piorou pouco (2), está igual (3), melhorou um pouco (4), melhorou muito (5). Os pontos foram somados em um índice que varia de 2 a 10, recodificados em três níveis: de 2 a 5 pontos como piorou, de 6 a 7 pontos como igual e de 8 a 10 como melhorou. [Criaram-se duas variáveis binárias: políticas públicas iguais (1) e outros valores (0) e políticas públicas melhores (1) e outros valores (0).]

2.2.2.5 Percepção do combate à corrupção: "o quanto você acredita que tem avançado o combate à corrupção?" nenhum progresso e pouco progresso (0), algum progresso e muito progresso (1).

\subsubsection{Variáveis sociodemográficas}

2.2.3.1 Grupos etários: até 25 anos de idade (grupo de referência); de 26 a 35 anos de idade (Seg. 26 a 35); de 36 a 45 anos de idade (Ter. 36 a 45); de 46 a 55 anos (Qua. 46 a 55); 56 anos de idade ou mais (Qui. ac.56).

\subsubsection{Sexo: mulher (0) e homem (1).}

2.2.3.3 Escolaridade: Analfabetos e Ensino Fundamental incompleto (grupo de referência), Ensino Fundamental completo, Ensino médio completo, Ensino Superior completo.

2.2.3.4 Nível socioeconômico, criado a partir das seguintes variáveis: 1 . posse de bens: posse de TV (0) não tem, (1) tem; posse de refrigerador (0) não tem, (1) tem; posse de casa própria (0) não tem, (1) tem; posse de máquina de lavar (0) não tem, (1) tem; posse de telefone (0) não tem, (1) tem; posse de celular (0) não tem, (1) tem; posse de computador (0) não tem, (2) tem; posse de carro (0) não tem, (2) 
tem; posse de casa de campo (0) não tem, (2) tem. 2. Educação dos pais: até fundamental incompleto (0), até fundamental completo (1), até médio completo (2), superior completo ou mais (3). [Os pontos foram somados em um índice que varia de 0 a 16 , recodificados em quatro níveis: de 0.3 muito baixo; 4.6 baixo; 7.11 médio; 12.16 alto.]

Atribuição de missing: Para o conjunto das variáveis independentes foi utilizado o sistema de imputação de valores (regressão) aos dados missing do pacote estatístico SPSS 13.0. Como demonstraram King et al (2001), os métodos tradicionais de exclusão (listwise e pairwise) e a atribuição de valores ausentes pela média dos valores disponíveis podem levar a estimativas ineficientes ou distorcidas. Por isso, os autores aconselham o uso da imputação de valores por métodos multivariados: os valores das variáveis conhecidas servindo para predizer o valor ausente.

Vale destacar que, após a atribuição imputação feita pelo SPSS, a partir das variáveis indicadas pelo pesquisador, a estrutura dos dados (média, variância e desvio padrão) é mantida bastante próxima da distribuição original. A avaliação da atribuição seguiu o critério da atribuição que mantivesse a estrutura da distribuição dos dados a mais próxima possível da distribuição original.

As variáveis empregadas como preditoras foram: anos de escolaridade dos pais variando de 1 a 17 anos; autoclassificação do indivíduo em uma escala de pobreza-riqueza; percepção da situação socioeconômica do respondente feita pelo entrevistador; grupos etários e sexo.

Célia Mara Ladeia Colen·colenc@terra.com.br

Recebido para publicação em dezembro de 2008.

Aprovado para publicação em agosto de 2009. 